\section{$\begin{array}{llllll}\text { I } & \mathbf{N} & \mathbf{F} & \mathbf{S} & \mathbf{Y} & \mathbf{S}\end{array}$} R E S E A R C H $\begin{array}{llllll}\mathbf{R} & \mathrm{E} & \mathrm{P} & \mathrm{O} & \mathrm{R} & \mathrm{T}\end{array}$

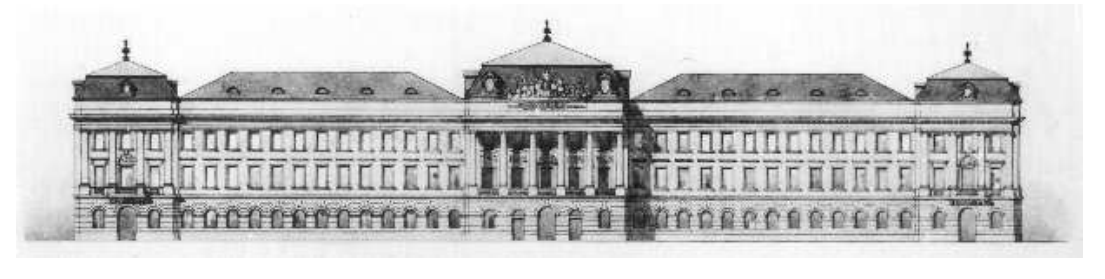

INSTITUT FÜR INFORMATIONSSYSTEME

ARbeitsbereich Wissensbasierte Systeme

\section{Tightly INTEGRATED FuZZY DesCRIPTION Logic PRograms Under THE ANSWER SET SEMANTICS For the SEMANTIC Web}

ThOMAS LUKASIEWICZ and UMBERTO STRACCIA
Institut für Informationssysteme AB Wissensbasierte Systeme Technische Universität Wien Favoritenstraße 9-11 A-1040 Wien, Austria Tel: $\quad+43-1-58801-18405$ Fax: +43-1-58801-18493 sek@kr.tuwien.ac.at www.kr.tuwien.ac.at
INFSYS RESEARCH REPORT 1843-07-03

FEBRUARY 2007

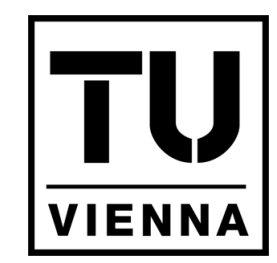



INFSYS RESEARCH REPORT

INFSYS RESEARCH REPORT 1843-07-03, FEBRUARY 2007

\title{
Tightly InTEgRATED FuZZY Description Logic PROGRAMS under the Answer Set Semantics for the Semantic Web
}

FEBRUARY 28, 2007

\author{
Thomas Lukasiewicz $^{1} \quad$ Umberto Straccia $^{2}$
}

\begin{abstract}
We present a novel approach to fuzzy dl-programs under the answer set semantics, which is a tight integration of fuzzy disjunctive programs under the answer set semantics with fuzzy description logics. From a different perspective, it is a generalization of tightly integrated disjunctive dl-programs by fuzzy vagueness in both the description logic and the logic program component. We show that the new formalism faithfully extends both fuzzy disjunctive programs and fuzzy description logics, and that under suitable assumptions, reasoning in the new formalism is decidable. Furthermore, we present a polynomial reduction of certain fuzzy dl-programs to tightly integrated disjunctive dl-programs. We also provide a special case of fuzzy dl-programs for which deciding consistency and query processing have both a polynomial data complexity.
\end{abstract}

\footnotetext{
${ }^{1}$ Dipartimento di Informatica e Sistemistica, Università di Roma "La Sapienza", Via Salaria 113, I-00198 Rome, Italy; e-mail: lukasiewicz@ dis.uniroma1.it. Institut für Informationssysteme, Technische Universität Wien, Favoritenstraße 9-11, A-1040 Vienna, Austria; e-mail: lukasiewicz@kr.tuwien.ac.at.

${ }^{2}$ ISTI-CNR, Via G. Moruzzi 1, I-56124 Pisa, Italy; e-mail: straccia@isti.cnr.it.
}

Acknowledgements: This work has been partially supported by a Heisenberg Professorship of the German Research Foundation (DFG).

Copyright (C) 2007 by the authors 


\section{Contents}

1 Introduction 1

2 Combination Strategies 2

3 Fuzzy Description Logics $\quad 2$

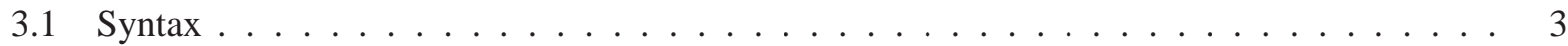

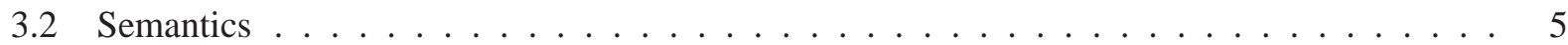

4 Fuzzy Description Logic Programs 6

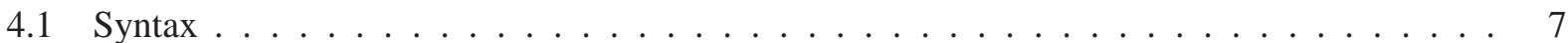

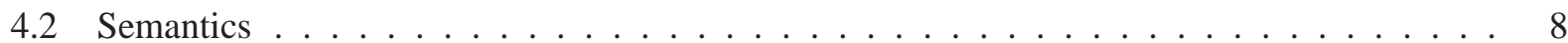

5 Semantic Properties 9

5.1 Minimal Models . . . . . . . . . . . . . . . . . . . . . . . . . . 10

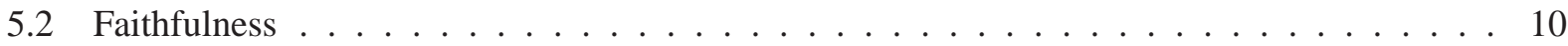

5.3 Unique Name Assumption . . . . . . . . . . . . . . . . . . . . . . . 10

6 Reduction of Fuzzy DL-Programs to DL-Programs 11

7 Tractability Results $\quad 12$

8 Summary and Outlook $\quad 13$ 


\section{Introduction}

The Semantic Web $[1,9]$ aims at an extension of the current World Wide Web by standards and technologies that help machines to understand the information on the Web so that they can support richer discovery, data integration, navigation, and automation of tasks. The main ideas behind it are to add a machine-readable meaning to Web pages, to use ontologies for a precise definition of shared terms in Web resources, to use KR technology for automated reasoning from Web resources, and to apply cooperative agent technology for processing the information of the Web.

The Semantic Web consists of several hierarchical layers, where the Ontology layer, in form of the OWL Web Ontology Language [34, 15], is currently the highest layer of sufficient maturity. OWL consists of three increasingly expressive sublanguages, namely, OWL Lite, OWL DL, and OWL Full. OWL Lite and OWL DL are essentially very expressive description logics with an RDF syntax [15]. As shown in [13], ontology entailment in OWL Lite (resp., OWL DL) reduces to knowledge base (un)satisfiability in the description logic $\mathcal{S H \mathcal { I }} \mathcal{F}(\mathbf{D})$ (resp., $\mathcal{S H O \mathcal { I }}(\mathbf{D})$ ). On top of the Ontology layer, the Rules, Logic, and Proof layers of the Semantic Web will be developed next, which should offer sophisticated representation and reasoning capabilities.

In particular, there is a large body of work on integrating rules and ontologies, which is a key requirement of the layered architecture of the Semantic Web. Significant research efforts focus on hybrid integrations of rules and ontologies, called description logic programs (or dl-programs), which are of the form $K B=(L, P)$, where $L$ is a description logic knowledge base and $P$ is a finite set of rules involving either queries to $L$ in a loose integration (see especially $[7,8,5,6]$ ) or concepts and roles from $L$ as unary resp. binary predicates in a tight integration (see especially $[25,26,20]$ ).

Other works explore formalisms for handling uncertainty and vagueness/imprecision in the Semantic Web. In particular, formalisms for dealing with uncertainty and vagueness in ontologies have been applied in ontology mapping and information retrieval. Vagueness and imprecision also abound in multimedia information processing and retrieval. Moreover, handling vagueness is an important aspect of natural language interfaces to the Web. There are several recent extensions of description logics, ontology languages, and description logic programs for the Semantic Web by probabilistic uncertainty and fuzzy vagueness. In particular, description logic programs under probabilistic uncertainty and fuzzy vagueness have been proposed in $[18,17]$ resp. $[31,32,19]$.

In this paper, we continue this line of research. We present tightly integrated fuzzy description logic programs (or simply fuzzy dl-programs) under the answer set semantics, which are a tight integration of fuzzy disjunctive programs under the answer set semantics with fuzzy generalizations of $\mathcal{S H \mathcal { F }} \mathcal{F}(\mathbf{D})$ and $\mathcal{S H O I N}(\mathbf{D})$. Even though there has been previous work on fuzzy positive dl-programs [31, 32] and on loosely integrated fuzzy normal dl-programs [19], to our knowledge, this is the first approach to tightly integrated fuzzy disjunctive dl-programs (with default negation in rule bodies). The main contributions of this paper can be summarized as follows:

- We present a novel approach to fuzzy dl-programs, which is a tight integration of fuzzy disjunctive programs under the answer set semantics with fuzzy description logics. It is a generalization of the tightly integrated disjunctive dl-programs in [20] by fuzzy vagueness in both the description logic and the logic program component.

- We show that the new fuzzy dl-programs have nice semantic features. In particular, all their answer sets are also minimal models, and the cautious answer set semantics faithfully extends both fuzzy disjunctive programs and fuzzy description logics. Furthermore, the new approach also does not need 
the unique name assumption.

- In the large class of fuzzy dl-programs that are defined over a finite number of truth values, the problems of deciding consistency, cautious consequence, and brave consequence are all decidable. We also present a polynomial reduction for certain fuzzy dl-programs to the tightly integrated disjunctive dl-programs in [20].

- Finally, we delineate a special case of fuzzy dl-programs where deciding consistency and query processing have both a polynomial data complexity.

The rest of this paper is organized as follows. Sections 2 and 3 recall combination strategies and fuzzy description logics, respectively. Section 4 introduces the syntax of fuzzy dl-programs and defines their answer set semantics. In Section 5, we analyze some semantic properties of fuzzy dl-programs under the answer set semantics. Section 6 presents a reduction of fuzzy dl-programs to disjunctive dl-programs. In Section 7, we delineate a special case of fuzzy dl-programs with polynomial data complexity. Section 8 summarizes our main results and gives an outlook on future research. Note that detailed proofs of all the results in this paper are given in the extended version.

\section{Combination Strategies}

Rather than being restricted to an ordinary binary truth value among false and true, vague propositions may also have a truth value strictly between false and true. In the sequel, we use the unit interval $[0,1]$ as the set of all possible truth values, where 0 and 1 represent the ordinary binary truth values false and true, respectively. For example, the vague proposition "John is a tall man" may be more or less true, and it is thus associated with a truth value in $[0,1]$, depending on the body height of John.

In order to combine and modify the truth values in $[0,1]$, we assume combination strategies, namely, conjunction, disjunction, implication, and negation strategies, denoted $\otimes, \oplus, \triangleright$, and $\ominus$, respectively, which are functions $\otimes, \oplus, \triangleright:[0,1] \times[0,1] \rightarrow[0,1]$ and $\ominus:[0,1] \rightarrow[0,1]$ that generalize the ordinary Boolean operators $\wedge, \vee, \rightarrow$, and $\neg$, respectively, to the set of truth values $[0,1]$. For $a, b \in[0,1]$, we then call $a \otimes b$ (resp., $a \oplus b, a \triangleright b$ ) the conjunction (resp., disjunction, implication) of $a$ and $b$, and we call $\ominus a$ the negation of $a$. As usual, we assume that combination strategies have some natural algebraic properties, namely, the properties shown in Tables 1 and 2. Note that conjunction and disjunction strategies (with the properties in Table 1) are also called triangular norms and triangular co-norms [11], respectively. We do not assume properties that relate the combination strategies to each other (such as de Morgan's law); even though one may additionally assume such properties, they are not required here.

Example 2.1 The combination strategies of various fuzzy logics are shown in Table 3.

\section{Fuzzy Description Logics}

In this section, we recall fuzzy $\mathcal{S H \mathcal { H }} \mathcal{F}(\mathbf{D})$ and fuzzy $\mathcal{S H O} \mathcal{H} \mathcal{N}(\mathbf{D})[29,30,21]$ (see also [27]). Note that there also exists an implementation of fuzzy $\mathcal{S H \mathcal { H }} \mathcal{F}(\mathbf{D})$ (the fuzzyDL system; see http: / / gaia. isti. cnr.it/ straccia). Intuitively, description logics model a domain of interest in terms of concepts and roles, which represent classes of individuals and binary relations between classes of individuals, respectively. A description logic knowledge base encodes in particular subset relationships between classes of individuals, 
Table 1: Axioms for conjunction and disjunction strategies.

\begin{tabular}{lll}
\hline Axiom Name & Conjunction Strategy & Disjunction Strategy \\
\hline Tautology / Contradiction & $a \otimes 0=0$ & $a \oplus 1=1$ \\
Identity & $a \otimes 1=a$ & $a \oplus 0=a$ \\
Commutativity & $a \otimes b=b \otimes a$ & $a \oplus b=b \oplus a$ \\
Associativity & $(a \otimes b) \otimes c=a \otimes(b \otimes c)$ & $(a \oplus b) \oplus c=a \oplus(b \oplus c)$ \\
Monotonicity & if $b \leqslant c$, then $a \otimes b \leqslant a \otimes c$ & if $b \leqslant c$, then $a \oplus b \leqslant a \oplus c$ \\
\hline
\end{tabular}

Table 2: Axioms for implication and negation strategies.

\begin{tabular}{lll}
\hline Axiom Name & Implication Strategy & Negation Strategy \\
\hline Tautology / Contradiction & $0 \triangleright b=1, a \triangleright 1=1,1 \triangleright 0=0$ & $\ominus 0=1, \ominus 1=0$ \\
Antitonicity & if $a \leqslant b$, then $a \triangleright c \geqslant b \triangleright c$ & if $a \leqslant b$, then $\ominus a \geqslant \ominus b$ \\
Monotonicity & if $b \leqslant c$, then $a \triangleright b \leqslant a \triangleright c$ & \\
\hline
\end{tabular}

Table 3: Combination strategies of various fuzzy logics.

\begin{tabular}{|c|c|c|c|c|}
\hline & Łukasiewicz Logic & Gödel Logic & Product Logic & Zadeh Logic \\
\hline$a \otimes b$ & $\max (a+b-1,0)$ & $\min (a, b)$ & $a \cdot b$ & $\min (a, b)$ \\
\hline$a \oplus b$ & $\min (a+b, 1)$ & $\max (a, b)$ & $a+b-a \cdot b$ & $\max (a, b)$ \\
\hline$a \triangleright b$ & $\min (1-a+b, 1)$ & $\begin{array}{ll}1 & \text { if } a \leqslant b \\
b & \text { otherwise }\end{array}$ & $\min (1, b / a)$ & $\max (1-a, b)$ \\
\hline$\ominus a$ & $1-a$ & $\begin{array}{ll}1 & \text { if } a=0 \\
0 & \text { otherwise }\end{array}$ & $\begin{cases}1 & \text { if } a=0 \\
0 & \text { otherwise }\end{cases}$ & $1-a$ \\
\hline
\end{tabular}

subset relationships between binary relations between classes, the membership of individuals to classes, and the membership of pairs of individuals to binary relations between classes. In fuzzy description logics, these relationships and memberships then have a degree of truth in $[0,1]$.

\subsection{Syntax}

We first describe fuzzy $\mathcal{S H O I N}(\mathbf{D})$, which has the following elementary ingredients. We assume a set of data values, a set of elementary datatypes, and a set of datatype predicates (each with a predefined arity $n \geqslant 1$ ). A datatype is an elementary datatype or a finite set of data values. A fuzzy datatype theory $\mathbf{D}=\left(\Delta^{\mathbf{D}},{ }^{\mathbf{D}}\right)$ consists of a datatype domain $\Delta^{\mathbf{D}}$ and a mapping ${ }^{\mathbf{D}}$ that assigns to each data value an element of $\Delta^{\mathrm{D}}$, to each elementary datatype a subset of $\Delta^{\mathrm{D}}$, and to each datatype predicate of arity $n$ a fuzzy relation over $\Delta^{\mathbf{D}}$ of arity $n$ (that is, a mapping $\left(\Delta^{\mathbf{D}}\right)^{n} \rightarrow[0,1]$ ). We extend ${ }^{\mathbf{D}}$ to all datatypes by $\left\{v_{1}, \ldots, v_{n}\right\}^{\mathbf{D}}=\left\{v_{1}^{\mathbf{D}}, \ldots, v_{n}^{\mathbf{D}}\right\}$.

Example 3.1 A crisp unary datatype predicate $\leqslant 18$ over the natural numbers denoting the integers of at most 18 may be defined by $\leqslant_{18}(x)=1$, if $x \leqslant 18$, and $\leqslant 18(x)=0$, otherwise. Then, Minor $=$ Person $\sqcap$ $\exists$ age. $\leqslant 18$ defines a person of age at most 18 . Non-crisp predicates are usually defined by functions for specifying fuzzy set membership degrees, such as the triangular, the trapezoidal, the $L$-, and the $R$ function (see Fig. 1). For example, a fuzzy unary datatype predicate Young over the natural numbers 


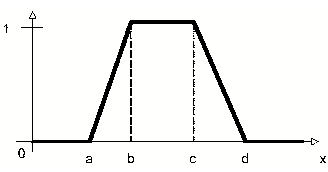

(a)

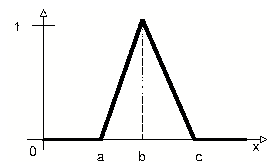

(b)

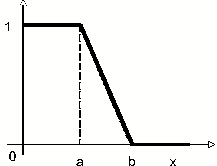

(c)

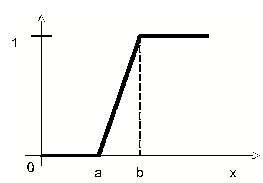

(d)

Figure 1: (a) Trapezoidal function; (b) Triangular function; (c) $L$-function; (d) $R$-function

denoting the degree of youngness of a person's age may be defined by Young $(x)=L(x ; 10,30)$. Then, YoungPerson $=$ Person $\sqcap \exists$ age. Young denotes a young person.

Let $\mathbf{A}, \mathbf{R}_{A}, \mathbf{R}_{D}, \mathbf{I}$, and $\mathbf{M}$ be pairwise disjoint (nonempty) denumerable sets of atomic concepts, abstract roles, datatype roles, individuals, and fuzzy modifiers, respectively. Here, a fuzzy modifier $m$ [12, 33] represents a function $f_{m}$ on $[0,1]$, which changes the membership function of a fuzzy set.

Example 3.2 The fuzzy modifiers very resp. slightly may represent the two functions very $(x)=x^{2}$ resp. $\operatorname{slightly}(x)=\sqrt{x}$. Then, the concept of sports cars may be defined as SportsCar $=$ Car $\sqcap \exists$ speed.very $($ High $)$, where High is a fuzzy datatype predicate over the domain of speed in $\mathrm{km} / \mathrm{h}$, which may be defined as $\operatorname{High}(x)=R(x ; 80,250)$.

A role is any element of $\mathbf{R}_{A} \cup \mathbf{R}_{A}^{-} \cup \mathbf{R}_{D}$ (where $\mathbf{R}_{A}^{-}$is the set of inverses $R^{-}$of all $R \in \mathbf{R}_{A}$ ). We define concepts inductively as follows. Each $A \in \mathbf{A}$ is a concept, $\perp$ and $\top$ are concepts, and if $a_{1}, \ldots$, $a_{n} \in \mathbf{I}$, then $\left\{a_{1}, \ldots, a_{n}\right\}$ is a concept (called oneOf). If $C, C_{1}, C_{2}$ are concepts, $R, S \in \mathbf{R}_{A} \cup \mathbf{R}_{A}^{-}$, and $m \in \mathbf{M}$, then $\left(C_{1} \sqcap C_{2}\right),\left(C_{1} \sqcup C_{2}\right), \neg C$, and $m(C)$ are concepts (called conjunction, disjunction, negation, and fuzzy modification, respectively), as well as $\exists R . C, \forall R . C, \geqslant n S$, and $\leqslant n S$ (called exists, value, atleast, and atmost restriction, respectively) for an integer $n \geqslant 0$. If $D$ is a datatype and $T, T_{1}, \ldots, T_{n} \in \mathbf{R}_{D}$, then $\exists T_{1}, \ldots, T_{n} . D, \forall T_{1}, \ldots, T_{n} . D, \geqslant n T$, and $\leqslant n T$ are concepts (called datatype exists, value, atleast, and atmost restriction, respectively) for an integer $n \geqslant 0$. We eliminate parentheses as usual.

A crisp axiom has one of the following forms: (1) $C \sqsubseteq D$ (called concept inclusion axiom), where $C$ and $D$ are concepts; (2) $R \sqsubseteq S$ (called role inclusion axiom), where either $R, S \in \mathbf{R}_{A}$ or $R, S \in \mathbf{R}_{D}$; (3) $\operatorname{Trans}(R)$ (called transitivity axiom), where $R \in \mathbf{R}_{A}$; (4) $C(a)$ (called concept assertion axiom), where $C$ is a concept and $a \in \mathbf{I}$; (5) $R(a, b)$ (resp., $U(a, v)$ ) (called role assertion axiom), where $R \in \mathbf{R}_{A}$ (resp., $U \in \mathbf{R}_{D}$ ) and $a, b \in \mathbf{I}$ (resp., $a \in \mathbf{I}$ and $v$ is a data value); and (6) $a=b$ (resp., $a \neq b$ ) (equality (resp., inequality) axiom), where $a, b \in \mathbf{I}$. We define fuzzy axioms as follows: A fuzzy concept inclusion (resp., fuzzy role inclusion, fuzzy concept assertion, fuzzy role assertion) axiom is of the form $\alpha \theta n$, where $\alpha$ is a concept inclusion (resp., role inclusion, concept assertion, role assertion) axiom, $\theta \in\{\leqslant,=, \geqslant\}$, and $n \in[0,1]$. For example, $C(a) \geqslant 0.1, R(a, b) \leqslant 0.3, R \sqsubseteq S \geqslant 0.4$, and $C \sqsubseteq D \leqslant 0.6$ are fuzzy axioms. Informally, a fuzzy axiom of the form $\alpha \leqslant n$ (resp., $\alpha=n, \alpha \geqslant n$ ) encodes that the truth degree of $\alpha$ is at most (resp., equal to, at least) $n$. For example, TallPerson $(\mathrm{jim}) \geqslant 0.2$ says that $\mathrm{jim}$ is a tall person with a truth degree of at least 0.2 , while $C \sqsubseteq D \geqslant n$ says that the subsumption degree between $C$ and $D$ is at least $n$. We often use $a$ : $C$ and $\alpha$ to abbreviate $C(a)$ and $\alpha \geqslant 1$, respectively. A fuzzy (description logic) knowledge base $L$ is a finite set of fuzzy axioms, transitivity axioms, and equality and inequality axioms. For decidability, number restrictions in $L$ are restricted to simple abstract roles [16].

Fuzzy $\mathcal{S H \mathcal { H }} \mathcal{F}(\mathbf{D})$ has the same syntax as fuzzy $\mathcal{S H O} \mathcal{H} \mathcal{N}(\mathbf{D})$, but without the oneOf constructor and with the atleast and atmost constructors limited to 0 and 1. 
Example 3.3 (Shopping Agent) The following axioms are an excerpt of the description logic knowledge base $L$ that conceptualizes a car selling web site:

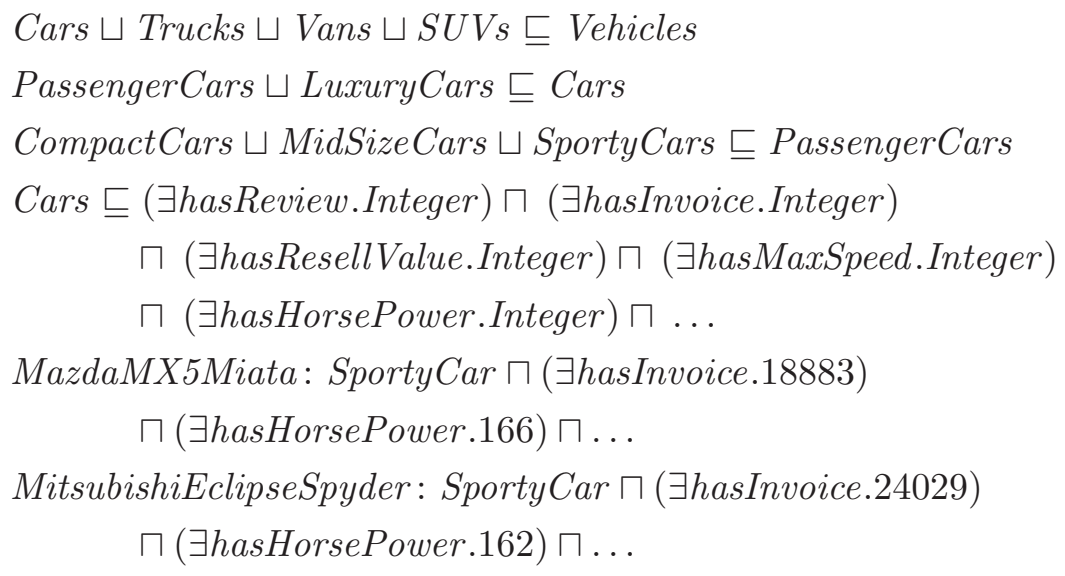

Eqs. 1-3 describe the concept taxonomy of the site, while Eq. 4 describes the datatype attributes of the cars sold in the site. Eqs. 5-6 describe the properties of some sold cars.

We may then encode "costs at most about $22000 €$ " and "has a power of around $150 \mathrm{HP}$ " in a buyer's request through the following concepts $C$ and $D$, respectively:

$$
C=\exists \text { hasInvoice.LeqAbout22000 and } D=\exists \text { hasHorsePower.Around150, }
$$

where LeqAbout22000 $=L(22000,25000)$ and Around150 $=\operatorname{Tr} i(125,150,175)$. The latter two equations define the fuzzy concepts of "at most about $22000 €$ " and "around 150 HP". The former is modeled as a left shoulder function stating that if the prize is less than 22000 , then the degree of truth (degree of buyer's satisfaction) is 1 , else the truth is linearly decreasing to 0 (reached at the cost of 25000). In fact, we are modeling a case were the buyer would like to pay less than 22000 , though may still accept a higher price (up to 25000 ) to a lesser degree. Similarly, the latter models the fuzzy concept "around $150 \mathrm{HP}$ " as a triangular function with vertice in $150 \mathrm{HP}$.

\subsection{Semantics}

Concerning the semantics of fuzzy $\mathcal{S H \mathcal { I }} \mathcal{F}(\mathbf{D})$ and $\mathcal{S H O \mathcal { N }}(\mathbf{D})$ [30], the main idea is that concepts and roles are interpreted as fuzzy subsets of an interpretation's domain. Therefore, concept inclusion, role inclusion, concept assertion, and role assertion axioms, rather than being satisfied (true) or unsatisfied (false) in an interpretation, have a degree of truth in $[0,1]$. In the sequel, we assume that $\otimes, \oplus, \triangleright$, and $\ominus$ are some arbitrary but fixed conjunction, disjunction, implication, and negation strategies, respectively. A fuzzy interpretation $\mathcal{I}=\left(\Delta^{\mathcal{I}},{ }^{\mathcal{I}}\right)$ relative to a fuzzy datatype theory $\mathbf{D}=\left(\Delta^{\mathbf{D}},{ }^{\mathbf{D}}\right)$ consists of a nonempty set $\Delta^{\mathcal{I}}$ (called the domain), disjoint from $\Delta^{\mathrm{D}}$, and a fuzzy interpretation function ${ }^{\mathcal{I}}$, which (i) coincides with . $\mathbf{D}$ on every data value, datatype, and fuzzy datatype predicate, (ii) assigns to each modifier $m \in \mathbf{M}$ its modifier function $f_{m}:[0,1] \rightarrow[0,1]$, and (iii) assigns

- to each individual $a \in \mathbf{I}$ an element $a^{\mathcal{I}} \in \Delta^{\mathcal{I}}$;

- to each atomic concept $C \in \mathbf{A}$ a function $C^{\mathcal{I}}: \Delta^{\mathcal{I}} \rightarrow[0,1]$;

- to each abstract role $R \in \mathbf{R}_{A}$ a function $R^{\mathcal{I}}: \Delta^{\mathcal{I}} \times \Delta^{\mathcal{I}} \rightarrow[0,1]$; 
- to each concrete role $T \in \mathbf{R}_{D}$ a function $T^{\mathcal{I}}: \Delta^{\mathcal{I}} \times \Delta^{\mathbf{D}} \rightarrow[0,1]$.

The mapping ${ }^{\mathcal{I}}$ is extended to all roles and concepts as follows (where $x, y \in \Delta^{\mathcal{I}}$ ):

$$
\begin{aligned}
& \begin{aligned}
\left(S^{-}\right)^{\mathcal{I}}(x, y) & =S^{\mathcal{I}}(y, x) \\
\top^{\mathcal{I}}(x) & =1
\end{aligned} \\
& \perp^{\mathcal{I}}(x)=0 \\
& \left\{a_{1}, \ldots, a_{n}\right\}^{\mathcal{I}}(x)=\bigoplus_{i=1}^{n} a_{i}{ }^{\mathcal{I}}=x \\
& \left(C_{1} \sqcap C_{2}\right)^{\mathcal{I}}(x)=C_{1}^{\mathcal{I}}(x) \otimes C_{2}^{\mathcal{I}}(x) \\
& \left(C_{1} \sqcup C_{2}\right)^{\mathcal{I}}(x)=C_{1}^{\mathcal{I}}(x) \oplus C_{2}^{\mathcal{I}}(x) \\
& (\neg C)^{\mathcal{I}}(x)=\ominus C^{\mathcal{I}}(x) \\
& (m(C))^{\mathcal{I}}(x)=f_{m}\left(C^{\mathcal{I}}(x)\right) \\
& (\forall R . C)^{\mathcal{I}}(x)=\inf _{y \in \Delta^{\mathcal{I}}} R^{\mathcal{I}}(x, y) \triangleright C^{\mathcal{I}}(y) \\
& (\exists R . C)^{\mathcal{I}}(x)=\sup _{y \in \Delta^{\mathcal{I}}} R^{\mathcal{I}}(x, y) \otimes C^{\mathcal{I}}(y)
\end{aligned}
$$

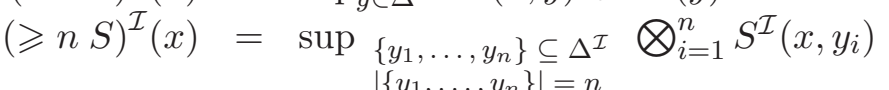

$$
\begin{aligned}
& (\leqslant n S)^{\mathcal{I}}(x)=\ominus(\geqslant n+1 S)^{\mathcal{I}}(x) \\
& \left(\forall T_{1}, \ldots, T_{n} . D\right)^{\mathcal{I}}(x)=\inf _{y_{1}, \ldots, y_{n} \in \Delta^{\mathrm{D}}}\left(\bigotimes_{i=1}^{n} T_{i}^{\mathcal{I}}\left(x, y_{i}\right)\right) \triangleright D^{\mathbf{D}}\left(y_{1}, \ldots, y_{n}\right) \\
& \left(\exists T_{1}, \ldots, T_{n} . D\right)^{\mathcal{I}}(x)=\sup _{y_{1}, \ldots, y_{n} \in \Delta^{\mathbf{D}}}\left(\bigotimes_{i=1}^{n} T_{i}^{\mathcal{I}}\left(x, y_{i}\right)\right) \otimes D^{\mathbf{D}}\left(y_{1}, \ldots, y_{n}\right) .
\end{aligned}
$$

The mapping $\cdot{ }^{\mathcal{I}}$ is extended to concept inclusion, role inclusion, concept assertion, and role assertion axioms as follows (where $a, b \in \mathbf{I}$ ):

$$
\begin{aligned}
\left(C_{1} \sqsubseteq C_{2}\right)^{\mathcal{I}} & =\inf _{x \in \Delta^{\mathcal{I}}} C_{1}{ }^{\mathcal{I}}(x) \triangleright C_{2}{ }^{\mathcal{I}}(x) \\
\left(R_{1} \sqsubseteq R_{2}\right)^{\mathcal{I}} & =\inf _{x, y \in \Delta^{\mathcal{I}}} R_{1}{ }^{\mathcal{I}}(x, y) \triangleright R_{2}{ }^{\mathcal{I}}(x, y) \\
(C(a))^{\mathcal{I}} & =C^{\mathcal{I}}\left(a^{\mathcal{I}}\right) \\
(R(a, b))^{\mathcal{I}} & =R^{\mathcal{I}}\left(a^{\mathcal{I}}, b^{\mathcal{I}}\right) .
\end{aligned}
$$

The notion of a fuzzy interpretation $\mathcal{I}$ satisfying a transitivity, equality, inequality, or fuzzy axiom $E$, or $\mathcal{I}$ being a model of $E$, denoted $\mathcal{I} \models E$, is defined as follows: (i) $\mathcal{I} \models \operatorname{trans}(R)$ iff $R^{\mathcal{I}}(x, y) \geqslant \sup _{z \in \Delta^{\mathcal{I}}}$ $R^{\mathcal{I}}(x, z) \otimes R^{\mathcal{I}}(z, y)$ for all $x, y \in \Delta^{\mathcal{I}}$; (ii) $\mathcal{I}=a=b$ iff $a^{\mathcal{I}}=b^{\mathcal{I}}$, and $\mathcal{I}=a \neq b$ iff $a^{\mathcal{I}} \neq b^{\mathcal{I}}$; and (iii) $\mathcal{I} \models$ $\alpha \theta n$ iff $\alpha^{\mathcal{I}} \theta n$. A concept $C$ is satisfiable iff there is an interpretation $\mathcal{I}$ and some $x \in \Delta^{\mathcal{I}}$ such that $C^{\mathcal{I}}(x)>0$. We say $\mathcal{I}$ satisfies a fuzzy knowledge base $L$, or $\mathcal{I}$ is a model of $L$, denoted $\mathcal{I} \models L$, iff $\mathcal{I}$ is a model of all $E \in L$. We say $L$ is satisfiable iff $L$ has a model. A fuzzy axiom $E$ is a logical consequence of $L$, denoted $L \models E$, iff every model of $L$ satisfies $E$. A fuzzy axiom $\alpha \geqslant n$ is a tight logical consequence of $L$, denoted $L=_{\text {tight }} \alpha \geqslant n$, iff $n$ is the supremum of $m \in[0,1]$ subject to $L \models \alpha \geqslant m$.

Example 3.4 (Shopping Agent cont'd) The following fuzzy axioms are (tight) logical consequences of $L$ in Example 3.3 (under the Zadeh semantics of the connectives):

$$
\begin{array}{ll}
C(\text { MazdaMX5Miata }) \geqslant 1.0 & C \text { (MitsubishiEclipseSpyder }) \geqslant 0.32 \\
D(\text { MazdaMX5Miata }) \geqslant 0.36 & \text { D(MitsubishiEclipseSpyder }) \geqslant 0.56 .
\end{array}
$$

\section{Fuzzy Description Logic Programs}

In this section, we present a tightly integrated approach to fuzzy disjunctive description logic programs (or simply fuzzy dl-programs) under the answer set semantics. Observe that differently from [19] (in addition 
to being a tightly integrated approach to fuzzy dl-programs), the fuzzy dl-programs here are based on fuzzy description logics as in [30]. Furthermore, they additionally allow for disjunctions in rule heads. We first introduce the syntax of fuzzy dl-programs and then their answer set semantics.

The basic idea behind the tightly integrated approach in this section is as follows. Suppose that we have a fuzzy disjunctive program $P$. Under the answer set semantics, $P$ is equivalent to its grounding ground $(P)$. Suppose now that some of the ground atoms in $\operatorname{ground}(P)$ are additionally related to each other by a fuzzy description logic knowledge base $L$. That is, some of the ground atoms in ground $(P)$ actually represent concept and role memberships relative to $L$. Thus, when processing ground $(P)$, we also have to consider $L$. However, we only want to do it to the extent that we actually need it for processing $\operatorname{ground}(P)$. Hence, when taking a fuzzy Herbrand interpretation $I \subseteq H B_{\Phi}$, we have to ensure that $I$ represents a valid truth value assignment relative to $L$. In other words, the main idea behind the semantics is to interpret $P$ relative to Herbrand interpretations that also satisfy $L$, while $L$ is interpreted relative to general interpretations over a first-order domain. Thus, we modularly combine the standard semantics of fuzzy disjunctive programs and of fuzzy description logics as in [19], which allows for building on the standard techniques and the results of both areas. However, our new approach here allows for a much tighter integration of $L$ and $P$.

\subsection{Syntax}

We assume a function-free first-order vocabulary $\Phi$ with nonempty finite sets of constant and predicate symbols. We use $\Phi_{c}$ to denote the set of all constant symbols in $\Phi$. We also assume pairwise disjoint (nonempty) denumerable sets $\mathbf{A}, \mathbf{R}_{A}, \mathbf{R}_{D}, \mathbf{I}$, and $\mathbf{M}$ of atomic concepts, abstract roles, datatype roles, individuals, and fuzzy modifiers, respectively, as in Section 3. We assume that $\Phi_{c}$ is a subset of $\mathbf{I}$. This assumption guarantees that every ground atom constructed from atomic concepts, abstract roles, datatype roles, and constants in $\Phi_{c}$ can be interpreted in the description logic component. We do not assume any other restriction on the vocabularies, that is, $\Phi$ and $\mathbf{A}$ (resp., $\mathbf{R}_{A} \cup \mathbf{R}_{D}$ ) may have unary (resp., binary) predicate symbols in common.

Let $\mathcal{X}$ be a set of variables. A term is either a variable from $\mathcal{X}$ or a constant symbol from $\Phi$. An atom is of the form $p\left(t_{1}, \ldots, t_{n}\right)$, where $p$ is a predicate symbol of arity $n \geqslant 0$ from $\Phi$, and $t_{1}, \ldots, t_{n}$ are terms. A literal $l$ is an atom $p$ or a negated atom not $p$. A disjunctive fuzzy rule (or simply fuzzy rule) $r$ is of the form

$$
\begin{aligned}
a_{1} \vee_{\oplus_{1}} \cdots \vee_{\oplus_{l-1}} a_{l} \leftarrow \otimes_{0} & b_{1} \wedge_{\otimes_{1}} b_{2} \wedge_{\otimes_{2}} \cdots \wedge_{\otimes_{k-1}} b_{k} \wedge_{\otimes_{k}} \\
& \operatorname{not}_{\ominus_{k+1}} b_{k+1} \wedge_{\otimes_{k+1}} \cdots \wedge_{\otimes_{m-1}} \text { not }_{\ominus_{m}} b_{m} \geqslant v,
\end{aligned}
$$

where $l \geqslant 1, m \geqslant k \geqslant 0, a_{1}, \ldots, a_{l}, b_{k+1}, \ldots, b_{m}$ are atoms, $b_{1}, \ldots, b_{k}$ are either atoms or truth values from $[0,1], \oplus_{1}, \ldots, \oplus_{l-1}$ are disjunction strategies, $\otimes_{0}, \ldots, \otimes_{m-1}$ are conjunction strategies, $\ominus_{k+1}, \ldots, \ominus_{m}$ are negation strategies, and $v \in[0,1]$. We refer to $a_{1} \vee_{\oplus_{1}} \cdots \vee_{\oplus_{l-1}} a_{l}$ as the head of $r$, while the conjunction $b_{1} \wedge_{\otimes_{1}} \ldots \wedge_{\otimes_{m-1}}$ not $_{\ominus_{m}} b_{m}$ is the body of $r$. We define $H(r)=\left\{a_{1}, \ldots, a_{l}\right\}$ and $B(r)=B^{+}(r) \cup B^{-}(r)$, where $B^{+}(r)=\left\{b_{1}, \ldots, b_{k}\right\}$ and $B^{-}(r)=\left\{b_{k+1}, \ldots, b_{m}\right\}$. A disjunctive fuzzy program (or simply fuzzy program $P$ is a finite set of fuzzy rules of the form (7). We say $P$ is a normal fuzzy program iff $l=1$ for all fuzzy rules (7) in $P$. We say $P$ is a positive fuzzy program iff $l=1$ and $m=k$ for all fuzzy rules (7) in $P$.

A disjunctive fuzzy description logic program (or simply fuzzy dl-program) $K B=(L, P)$ consists of a description logic knowledge base $L$ and a disjunctive fuzzy program $P$. It is called a normal fuzzy dlprogram iff $P$ is a normal fuzzy program. It is called a positive fuzzy dl-program iff $P$ is a positive fuzzy program. 
Example 4.1 (Shopping Agent cont'd) A fuzzy dl-program $K B=(L, P)$ is given by the fuzzy description logic knowledge base $L$ in Example 3.3 and the set of fuzzy dl-rules $P$, which contains only the following fuzzy dl-rule (where $x \otimes y=\min (x, y)$ ):

$$
\begin{aligned}
\operatorname{query}(x) \leftarrow \leftarrow_{\otimes} & \text { SportyCar }(x) \wedge_{\otimes} \text { hasInvoice }\left(x, y_{1}\right) \wedge_{\otimes} \text { hasHorsePower }\left(x, y_{2}\right) \wedge_{\otimes} \\
& \text { LeqAbout22000 }\left(y_{1}\right) \wedge_{\otimes} \text { Around150 }\left(y_{2}\right) \geqslant 1 .
\end{aligned}
$$

Informally, the predicate query collects all sporty cars, and ranks them according to whether they cost at most around $22000 €$ and have around $150 \mathrm{HP}$ (such a car may be requested by a car buyer with economic needs). Another fuzzy dl-rule is given as follows (where $\ominus x=1-x$ and $\operatorname{Around} 300=\operatorname{Tr} i(250,300,350)$ ):

$$
\begin{aligned}
\text { query }^{\prime}(x) \leftarrow_{\otimes} & \text { SportyCar }(x) \wedge_{\otimes} \text { hasInvoice }\left(x, y_{1}\right) \wedge_{\otimes} \operatorname{hasMaxSpeed}\left(x, y_{2}\right) \wedge_{\otimes} \\
& \operatorname{not}_{\ominus} \operatorname{LeqAbout22000}\left(y_{1}\right) \wedge_{\otimes} \operatorname{Around} 300\left(y_{2}\right) \geqslant 1 .
\end{aligned}
$$

Informally, this rule collects all sporty cars, and ranks them according to whether they cost at least around $22000 €$ and have a maximum speed of around $300 \mathrm{~km} / \mathrm{h}$ (such a car may be requested by a car buyer with luxurious needs). Another fuzzy dl-rule involving also a disjunction in its head is given as follows (where $x \oplus y=\max (x, y))$ :

$$
\operatorname{Small}(x) \vee_{\oplus} \operatorname{Old}(x) \leftarrow_{\otimes} \operatorname{Car}(x) \wedge_{\otimes} \operatorname{hasInvoice}(x, y) \wedge_{\otimes} \operatorname{not}_{\ominus} \operatorname{GeqAbout15000}(y) \geqslant 0.7 .
$$

This rule says that a car costing at most around $15000 €$ is either small or old. Observe here that Small and Old may be two concepts in the fuzzy description logic knowledge base $L$. That is, the tightly integrated approach to fuzzy dl-programs under the answer set semantics also allows for using the rules in $L$ to express relationships between the concepts and roles in $P$. This is not possible in the loosely integrated approach to fuzzy dl-programs under the answer set semantics in [19], since the dl-queries of that framework can only occur in rule bodies, but not in rule heads.

\subsection{Semantics}

We now define the answer set semantics of fuzzy dl-programs via a generalization of the standard GelfondLifschitz transformation [10].

In the sequel, let $K B=(L, P)$ be a fuzzy dl-program. A ground instance of a rule $r \in P$ is obtained from $r$ by replacing every variable that occurs in $r$ by a constant symbol from $\Phi_{c}$. We denote by ground $(P)$ the set of all ground instances of rules in $P$. The Herbrand base relative to $\Phi$, denoted $H B_{\Phi}$, is the set of all ground atoms constructed with constant and predicate symbols from $\Phi$. Observe that we define the Herbrand base relative to $\Phi$ and not relative to $P$. This allows for reasoning about ground atoms from the description logic component that do not necessarily occur in $P$. Observe, however, that the extension from $P$ to $\Phi$ is only a notational simplification, since we can always make constant and predicate symbols from $\Phi$ occur in $P$ by "dummy" rules such as constant $(c) \leftarrow$ and $p(\boldsymbol{c}) \leftarrow p(\boldsymbol{c})$, respectively. We denote by $D L_{\Phi}$ the set of all ground atoms in $H B_{\Phi}$ that are constructed from atomic concepts in $\mathbf{A}$, abstract roles in $\mathbf{R}_{A}$, concrete roles in $\mathbf{R}_{D}$, and constant symbols in $\Phi_{c}$.

We define Herbrand interpretations and the truth of fuzzy dl-programs in them as follows. An interpretation $I$ is a mapping $I: H B_{\Phi} \rightarrow[0,1]$. We write $\boldsymbol{H B}_{\Phi}$ to denote the interpretation $I$ such that $I(a)=1$ for all $a \in H B_{\Phi}$. For interpretations $I$ and $J$, we write $I \subseteq J$ iff $I(a) \leqslant J(a)$ for all $a \in H B_{\Phi}$, and we define 
the intersection of $I$ and $J$, denoted $I \cap J$, by $(I \cap J)(a)=\min (I(a), J(a))$ for all $a \in H B_{\Phi}$. Observe that $I \subseteq \boldsymbol{H} \boldsymbol{B}_{\Phi}$ for all interpretations $I$. We say that $I$ is a model of a ground fuzzy rule $r$ of the form (7), denoted $I \models r$, iff

$$
\begin{aligned}
I\left(a_{1}\right) \oplus_{1} \cdots \oplus_{l} I\left(a_{l}\right) \geqslant & I\left(b_{1}\right) \otimes_{1} \cdots \otimes_{k-1} I\left(b_{k}\right) \otimes_{k} \\
& \ominus_{k+1} I\left(b_{k+1}\right) \otimes_{k+1} \cdots \otimes_{m-1} \ominus_{m} I\left(b_{m}\right) \otimes_{0} v .
\end{aligned}
$$

Here, we implicitly assume that the disjunction strategies $\oplus_{1}, \ldots, \oplus_{l}$ and the conjunction strategies $\otimes_{1}, \ldots$, $\otimes_{m-1}, \otimes_{0}$ are evaluated from left to right. Notice also that the above definition implicitly assumes an implication strategy $\triangleright$ that is defined by $a \triangleright b=\sup \left\{c \in[0,1] \mid a \otimes_{0} c \leqslant b\right\}$ for all $a, b \in[0,1]$ (and thus for $n, m \in[0,1]$ and $a=n$, it holds that $a \triangleright b \geqslant m$ iff $b \geqslant n \otimes_{0} m$, if we assume that the conjunction strategy $\otimes_{0}$ is continuous). Observe that such a relationship between the implication strategy $\triangleright$ and the conjunction strategy $\otimes$ (including also the continuity of $\otimes$ ) holds in Łukasiewicz, Gödel, and Product Logic (see Table 3). We say that $I$ is a model of a fuzzy program $P$, denoted $I \models P$, iff $I \models r$ for all $r \in \operatorname{ground}(P)$. We say $I$ is a model of a description logic knowledge base $L$, denoted $I=L$, iff $L \cup\left\{a=I(a) \mid a \in H B_{\Phi}\right\}$ is satisfiable. An interpretation $I \subseteq H B_{\Phi}$ is a model of a fuzzy dl-program $K B=(L, P)$, denoted $I \models K B$, iff $I=L$ and $I \models P$. We say $K B$ is satisfiable iff it has a model.

The Gelfond-Lifschitz transform of a fuzzy dl-program $K B=(L, P)$ relative to an interpretation $I \subseteq$ $\boldsymbol{H} \boldsymbol{B}_{\Phi}$, denoted $K B^{I}$, is defined as the fuzzy dl-program $\left(L, P^{I}\right)$, where $P^{I}$ is the set of all fuzzy rules obtained from $\operatorname{ground}(P)$ by replacing all default-negated atoms $\operatorname{not}_{\ominus_{j}} b_{j}$ by the truth value $\ominus_{j} I\left(b_{j}\right)$. We are now ready to define the answer set semantics of fuzzy dl-programs as follows.

Definition 4.2 Let $K B=(L, P)$ be a fuzzy dl-program. An interpretation $I \subseteq \boldsymbol{H B}_{\Phi}$ is an answer set of $K B$ iff $I$ is a minimal model of $K B^{I}$. We say that $K B$ is consistent (resp., inconsistent) iff $K B$ has an (resp., no) answer set.

We finally define the notions of cautious (resp., brave) reasoning from fuzzy dl-programs under the answer set semantics as follows.

Definition 4.3 Let $K B=(L, P)$ be a fuzzy dl-program. Let $a \in H B_{\Phi}$ and $n \in[0,1]$. Then, $a \geqslant n$ is a cautious (resp., brave) consequence of a fuzzy dl-program $K B$ under the answer set semantics iff $I(a) \geqslant n$ for every (resp., some) answer set $I$ of $K B$.

Example 4.4 (Shopping Agent cont'd) Consider again the fuzzy dl-program $K B=(L, P)$ of Example 4.1. The following holds for the answer set $M$ of $K B$ :

$$
M(q(\text { MazdaMX5Miata }))=0.36 \quad M(q(\text { MitsubishiEclipseSpyder }))=0.32 .
$$

\section{Semantic Properties}

In this section, we summarize some semantic properties (especially those relevant for the Semantic Web) of fuzzy dl-programs under the above answer set semantics. 


\subsection{Minimal Models}

The following theorem shows that, like for ordinary disjunctive programs, every answer set of a fuzzy dlprogram $K B$ is also a minimal model of $K B$, and the answer sets of a positive fuzzy dl-program $K B$ are the minimal models of $K B$.

Theorem 5.1 Let $K B=(L, P)$ be a fuzzy dl-program. Then, (a) every answer set of $K B$ is a minimal model of $K B$, and $(b)$ if $K B$ is positive, then the set of all answer sets of $K B$ is given by the set of all minimal models of $K B$.

\subsection{Faithfulness}

An important property of integrations of rules and ontologies is that they are a faithful $[22,23]$ extension of both rules and ontologies.

The following theorem shows that the answer set semantics of fuzzy dl-programs faithfully extends its counterpart for fuzzy programs. That is, the answer set semantics of a fuzzy dl-program $K B=(L, P)$ with empty fuzzy description logic knowledge base $L$ coincides with the answer set semantics of its fuzzy program $P$.

Theorem 5.2 Let $K B=(L, P)$ be a fuzzy dl-program such that $L=\emptyset$. Then, the set of all answer sets of $K B$ coincides with the set of all answer sets of the fuzzy program $P$.

The next theorem shows that the answer set semantics of fuzzy dl-programs also faithfully extends the first-order semantics of fuzzy description logic knowledge bases. That is, for $a \in H B_{\Phi}$ and $n \in[0,1]$, it holds that $a \geqslant n$ is true in all answer sets of a positive fuzzy dl-program $K B=(L, P)$ iff $a \geqslant n$ is true in all fuzzy first-order models of $L \cup \operatorname{ground}(P)$. The theorem holds also when $a$ is a ground formula constructed from $H B_{\Phi}$ using $\wedge$ and $\vee$, along with conjunction and disjunction strategies $\otimes$ resp. $\oplus$.

Theorem 5.3 Let $K B=(L, P)$ be a positive fuzzy dl-program, and let $a \in H B_{\Phi}$ and $n \in[0,1]$. Then, $a \geqslant n$ is true in all answer sets of $K B$ iff $a \geqslant n$ is true in all fuzzy first-order models of $L \cup \operatorname{ground}(P)$.

As an immediate corollary, we obtain that $a \geqslant n$ is true in all answer sets of a fuzzy dl-program $K B=$ $(L, \emptyset)$ iff $a \geqslant n$ is true in all fuzzy first-order models of $L$.

Corollary 5.4 Let $K B=(L, P)$ be a fuzzy dl-program with $P=\emptyset$, and let $a \in H B_{\Phi}$ and $n \in[0,1]$. Then, $a \geqslant n$ is true in all answer sets of $K B$ iff $a \geqslant n$ is true in all fuzzy first-order models of $L$.

\subsection{Unique Name Assumption}

Another aspect that may not be very desirable in the Semantic Web [14] is the unique name assumption (which says that any two distinct constant symbols in $\Phi_{c}$ represent two distinct domain objects). It turns out that we actually do not have to make this assumption, since the fuzzy description logic knowledge base of a fuzzy dl-program may very well contain or imply equalities between individuals.

This result is included in the following theorem, which shows an alternative characterization of the satisfaction of $L$ in $I \subseteq \boldsymbol{H B}_{\Phi}$ : Rather than being enlarged by a set of axioms of exponential size, $L$ is enlarged by a set of axioms of polynomial size. This characterization essentially shows that the satisfaction of $L$ in $I$ corresponds to checking that (i) $I$ restricted to $D L_{\Phi}$ satisfies $L$, and (ii) $I$ restricted to $H B_{\Phi}-D L_{\Phi}$ 
does not violate any equality axioms that follow from $L$. In the theorem, an equivalence relation $\sim$ on $\Phi_{c}$ is admissible with an interpretation $I \subseteq \boldsymbol{H B}_{\Phi}$ iff $I\left(p\left(c_{1}, \ldots, c_{n}\right)\right)=I\left(p\left(c_{1}^{\prime}, \ldots, c_{n}^{\prime}\right)\right)$ for all $n$-ary predicate symbols $p$, where $n>0$, and constant symbols $c_{1}, \ldots, c_{n}, c_{1}^{\prime}, \ldots, c_{n}^{\prime} \in \Phi_{c}$ such that $c_{i} \sim c_{i}^{\prime}$ for all $i \in\{1, \ldots, n\}$.

Theorem 5.5 Let $L$ be a fuzzy description logic knowledge base, and let $I \subseteq \boldsymbol{H B}_{\Phi}$. Then, $L \cup\{a=I(a) \mid$ $\left.a \in H B_{\Phi}\right\}$ is satisfiable iff $L \cup\left\{a=I(a) \mid a \in D L_{\Phi}\right\} \cup\left\{c \neq c^{\prime} \mid c \not c^{\prime}\right\}$ is satisfiable for some equivalence relation $\sim$ on $\Phi_{c}$ admissible with $I$.

\section{Reduction of Fuzzy DL-Programs to DL-Programs}

In this section, we present a polynomial reduction of fuzzy dl-programs to the tightly integrated dl-programs in [20]. Hence, reasoning in fuzzy dl-programs under the answer set semantics can be reduced to (a) reasoning in tightly integrated dl-programs under the answer set semantics and (b) reasoning in fuzzy description logics. Note that reasoning in fuzzy description logics may additionally be reduced to reasoning in crisp description logics along the lines presented in $[28,2]$ for fuzzy $\mathcal{A L C H}$ and fuzzy $\mathcal{S H O \mathcal { N }}$.

The reduction applies to all fuzzy dl-programs $K B$ that (i) are closed under $T V_{n}=\left\{0, \frac{1}{n}, \ldots, \frac{n}{n}\right\}$ for some $n>0$ and (ii) contain only combination strategies from Zadeh Logic. Here, KB is closed under $T V_{n}$ iff (a) every datatype predicate in $K B$ is interpreted by a mapping to $T V_{n}$, (b) every fuzzy modifier $m$ in $K B$ is interpreted by a mapping $f_{m}: T V_{n} \rightarrow T V_{n}$, (c) every truth value in $K B$ is from $T V_{n}$, and (d) every combination strategy in $K B$ is closed under $T V_{n}$ (which holds, e.g., for the combination strategies of Łukasiewicz, Gödel, and Zadeh Logic). Note that for fuzzy dl-programs $K B$ that are closed under $T V_{n}$, the problems of deciding consistency, cautious consequences, and brave consequences are all decidable, since we only have to consider the finite number of interpretations $I \subseteq \boldsymbol{H B}_{\Phi}$ that map to $T V_{n}$.

We denote by $\Phi^{n}$ the alphabet that is obtained from the alphabet $\Phi$ by replacing every predicate symbol $p$ by the new predicate symbols $p^{\alpha}$ with $\alpha \in T V_{n}^{+}=T V_{n} \backslash\{0\}$. For atoms $a=p\left(t_{1}, \ldots, t_{k}\right)$ and $\alpha \in T V_{n}^{+}$, the atom $a^{\alpha}$ over $\Phi^{n}$ is defined by $a^{\alpha}=p^{\alpha}\left(t_{1}, \ldots, t_{k}\right)$. Every fuzzy interpretation $I \subseteq \boldsymbol{H} \boldsymbol{B}_{\Phi}$ is associated with the binary interpretation $t(I)=\left\{a^{\alpha} \mid a \in H B_{\Phi}, \alpha \in T V_{n}^{+}, I(a) \geqslant \alpha\right\}$.

The crisp transform of a fuzzy dl-program $K B=(L, P)$ is the dl-program $t(K B)=(L, t(P))$, where $t(P)$ is the set (i) of all rules $p^{\beta}\left(x_{1}, \ldots, x_{k}\right) \leftarrow p^{\alpha}\left(x_{1}, \ldots, x_{k}\right)$ such that $p$ is a $k$-ary predicate symbol from $\Phi, x_{1}, \ldots, x_{k}$ are distinct variables, $\alpha \in T V_{n}^{+} \backslash\left\{\frac{1}{n}\right\}$, and $\beta=\alpha-\frac{1}{n}$, and (ii) of all rules $a_{1}^{\alpha} \vee \cdots \vee$ $a_{l}^{\alpha} \leftarrow b_{1}^{\alpha} \wedge \cdots \wedge b_{k}^{\alpha} \wedge$ not $b_{k+1}^{\gamma} \wedge \cdots \wedge$ not $b_{m}^{\gamma}$ such that a rule of the form (7) belongs to $P, \alpha \in T V_{n}^{+}, \alpha \leqslant v$, and $\gamma=1-\alpha+\frac{1}{n}$. Observe here that the generated dl-program $t(P)$ has a polynomial size in $P$ and $T V_{n}^{+}$ (assuming a unary number encoding for the truth values). The following theorem shows that the answer sets of $K B$ correspond to the answer sets of $t(K B)$ as in [20].

Theorem 6.1 Let $K B=(L, P)$ be a fuzzy dl-program that $(i)$ is closed under $T V_{n}=\left\{0, \frac{1}{n}, \ldots, \frac{n}{n}\right\}$ for some $n>0$ and (ii) contains only combination strategies from Zadeh Logic. Then, $I \subseteq \boldsymbol{H B}_{\Phi}$ is an answer set of $K B$ iff $t(I)$ is an answer set of $t(K B)$.

Example 6.2 (Shopping Agent cont'd) The last fuzzy dl-rule of Example 4.1 is translated into the following dl-rules in the crisp transform (for $T V_{10}=\{0,0.1, \ldots, 1\}$ ):

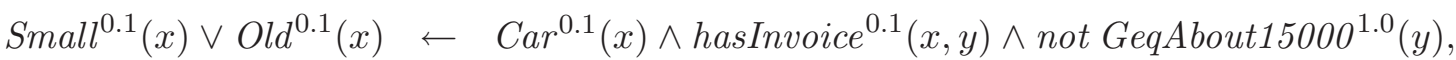

$$
\begin{aligned}
& \operatorname{Small}^{0.2}(x) \vee \operatorname{Old}^{0.2}(x) \leftarrow \operatorname{Car}^{0.2}(x) \wedge \text { hasInvoice }^{0.2}(x, y) \wedge \text { not GeqAbout15000 }{ }^{0.9}(y),
\end{aligned}
$$




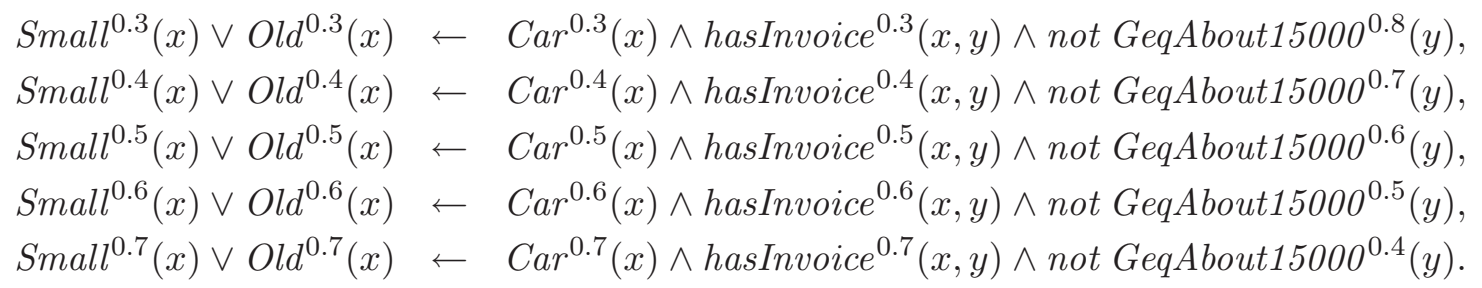

\section{Tractability Results}

In this section, we present a special class of fuzzy dl-programs $K B$ for which the problems of deciding consistency and of query processing have both a polynomial data complexity. These fuzzy dl-programs are defined relative to fuzzy DL-Lite [32], which is a fuzzy generalization of the description logic DL-Lite [4]. By [32] (resp., [4]), deciding whether a knowledge base in DL-Lite (resp., fuzzy DL-Lite) is satisfiable can be done in polynomial time, and conjunctive query processing from a knowledge base in DL-Lite (resp., fuzzy DL-Lite) has a polynomial data complexity.

We first recall DL-Lite and fuzzy DL-Lite. Let $\mathbf{A}, \mathbf{R}_{A}$, and $\mathbf{I}$ be pairwise disjoint sets of atomic concepts, abstract roles, and individuals, respectively. A basic concept in fuzzy DL-Lite is either an atomic concept from A or an exists restriction on roles $\exists R$. $\top$ (abbreviated as $\exists R$ ), where $R \in \mathbf{R}_{A} \cup \mathbf{R}_{A}^{-}$. A literal in DL-Lite is either a basic concept $b$ or the negation of a basic concept $\neg b$. Concepts in DL-Lite are defined by induction as follows. Every basic concept in DL-Lite is a concept in DL-Lite. If $b$ is a basic concept in DL-Lite, and $\phi_{1}$ and $\phi_{2}$ are concepts in DL-Lite, then $\neg b$ and $\phi_{1} \sqcap \phi_{2}$ are also concepts in DL-Lite. An axiom in DL-Lite is either (1) a concept inclusion axiom $b \sqsubseteq \psi$, where $b$ is a basic concept in DL-Lite, and $\phi$ is a concept in DL-Lite, or (2) a functionality axiom (funct $R$ ), where $R \in \mathbf{R}_{A} \cup \mathbf{R}_{A}^{-}$, or (3) a concept assertion axiom $b(a)$, where $b$ is a basic concept in DL-Lite and $a \in \mathbf{I}$, or (4) a role assertion axiom $R(a, c)$, where $R \in \mathbf{R}_{A}$ and $a, c \in \mathbf{I}$. A fuzzy concept (resp., role) assertion axiom is of the form $b(a) \geqslant n$ (resp., $R(a, c) \geqslant n$ ), where $b(a)$ (resp., $R(a, c)$ ) is a concept (resp., role) assertion axiom in DL-Lite, and $n \in[0,1]$. A fuzzy axiom in $D L$-Lite is either a fuzzy concept assertion axiom or a fuzzy role assertion axiom. A fuzzy knowledge base in DL-Lite $L$ is a finite set of concept inclusion, functionality, fuzzy concept assertion, and fuzzy role assertion axioms in DL-Lite.

For the conjunction strategies of Gödel and Zadeh Logic, every knowledge base in fuzzy DL-Lite L can be transformed into an equivalent one in fuzzy DL-Lite $\operatorname{trans}(L)$ in which every concept inclusion axiom is of form $b \sqsubseteq \ell$, where $b$ (resp., $\ell$ ) is a basic concept (resp., literal) in DL-Lite. We then define $\operatorname{trans}(K B)=(L, \operatorname{trans}(P))$ by $\operatorname{trans}(P)=P \cup\left\{b^{\prime}(X) \leftarrow b(X) \mid b \sqsubseteq b^{\prime} \in \operatorname{trans}(L), b^{\prime}\right.$ is a basic concept $\} \cup$ $\left\{\exists R(X) \leftarrow R(X, Y) \mid R \in \mathbf{R}_{A} \cap \Phi\right\} \cup\left\{\exists R^{-}(Y) \leftarrow R(X, Y) \mid R \in \mathbf{R}_{A} \cap \Phi\right\}$.

We are now ready to define fuzzy dl-programs in $D L$-Lite as follows. We say that a fuzzy dl-program $K B=(L, P)$ is defined in DL-Lite iff (i) $L$ is in fuzzy DL-Lite and interpreted relative to the conjunction strategies of Gödel or Zadeh Logic, (ii) $\operatorname{trans}(P)$ is normal and locally stratified, and (iii) $K B$ is closed under $T V_{n}=\left\{0, \frac{1}{n}, \ldots, \frac{n}{n}\right\}$ for some $n>0$, where we assume a unary encoding of the numbers in $T V_{n}$.

It can be shown that fuzzy dl-programs in DL-Lite have either no or a unique answer set, which can be computed by a finite sequence of fixpoint iterations, as usual. This implies immediately that for such programs, consistency checking and query processing have both a polynomial data complexity, which is formally expressed as follows. 
Theorem 7.1 Let $K B=(L, P)$ be a fuzzy dl-program in DL-Lite. Then, (a) deciding whether KB has an answer set, and (b) computing the truth value of a ground atom $a \in H B_{\Phi}$ in the answer set of $K B$ have both a polynomial data complexity.

\section{Summary and Outlook}

We have presented an approach to tightly integrated fuzzy dl-programs under the answer set semantics, which generalizes the tightly integrated disjunctive dl-programs in [20] by fuzzy vagueness in both the description logic and the logic program component. We have shown that the new formalism faithfully extends both fuzzy disjunctive programs and fuzzy description logics, and that under suitable assumptions, reasoning in the new formalism is decidable. Furthermore, we have presented a polynomial reduction for certain fuzzy dl-programs to tightly integrated disjunctive dl-programs. Finally, we have also provided a special case of fuzzy dl-programs for which deciding consistency and query processing have both a polynomial data complexity.

An interesting topic for future research is to analyze the computational complexity of the main reasoning problems in fuzzy dl-programs, and to implement the approach. Another interesting issue is to extend fuzzy dl-programs by classical negation.

\section{References}

[1] T. Berners-Lee. Weaving the Web. Harper, San Francisco, 1999.

[2] F. Bobillo, M. Delgado, and J. Gómez-Romero. A crisp representation for fuzzy $\mathcal{S H O I N}$ with fuzzy nominals and general concept inclusions. In Proc. URSW-2006, 2006.

[3] A. Calì and T. Lukasiewicz. An approach to probabilistic data integration for the Semantic Web. In Proc. URSW-2006, 2006.

[4] D. Calvanese, G. De Giacomo, D. Lembo, M. Lenzerini, and R. Rosati. DL-Lite: Tractable description logics for ontologies. In Proc. AAAI-2005, pp. 602-607, 2005.

[5] T. Eiter, G. Ianni, R. Schindlauer, H. Tompits. A uniform integration of higher-order reasoning and external evaluations in answer-set programming. In Proc. IJCAI-2005, 2005.

[6] T. Eiter, G. Ianni, R. Schindlauer, H. Tompits. Effective integration of declarative rules with external evaluations for Semantic Web reasoning. In Proc. ESWC-2006, pp. 273-287, 2006.

[7] T. Eiter, T. Lukasiewicz, R. Schindlauer, and H. Tompits. Combining answer set programming with description logics for the Semantic Web. In Proc. KR-2004, pp. 141-151, 2004.

[8] T. Eiter, T. Lukasiewicz, R. Schindlauer, and H. Tompits. Well-founded semantics for description logic programs in the Semantic Web. In Proc. RuleML-2004, pp. 81-97, 2004.

[9] D. Fensel, W. Wahlster, H. Lieberman, and J. Hendler, editors. Spinning the Semantic Web: Bringing the World Wide Web to Its Full Potential. MIT Press, 2002.

[10] M. Gelfond and V. Lifschitz. Classical negation in logic programs and disjunctive databases. New Generation Comput., 9(3/4):365-386, 1991. 
[11] P. Hájek. Metamathematics of Fuzzy Logic. Kluwer, 1998.

[12] S. Hölldobler, H.-P. Störr, and T. D. Khang. The subsumption problem of the fuzzy description logic $A L C_{F H}$. In Proc. IPMU-2004, pp. 243-250, 2004.

[13] I. Horrocks and P. F. Patel-Schneider. Reducing OWL entailment to description logic satisfiability. In Proc. ISWC-2003, pp. 17-29, 2003.

[14] I. Horrocks and P. F. Patel-Schneider. Position paper: A comparison of two modelling paradigms in the Semantic Web. In Proc. WWW-2006, pp. 3-12, 2006.

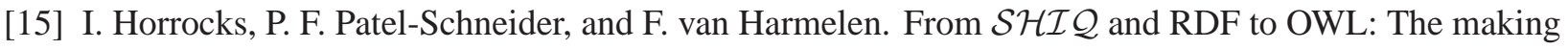
of a web ontology language. J. Web Sem., 1(1):7-26, 2003.

[16] I. Horrocks, U. Sattler, and S. Tobies. Practical reasoning for expressive description logics. In Proc. LPAR-1999, pp. 161-180, 1999.

[17] T. Lukasiewicz. Stratified probabilistic description logic programs. In Proc. URSW-2005, pp. 87-97, 2005.

[18] T. Lukasiewicz. Probabilistic description logic programs. In Proc. ECSQARU-2005, pp. 737-749, 2005. Extended version in Int. J. Approx. Reason., 2007 (in press).

[19] T. Lukasiewicz. Fuzzy description logic programs under the answer set semantics for the Semantic Web. In Proc. RuleML-2006, pp. 89-96, 2006.

[20] T. Lukasiewicz. A novel combination of answer set programming with description logics for the Semantic Web. Report INFSYS RR-1843-06-08, Institut für Informationssysteme, TU Wien, Dec. 2006. Available at http://www.kr.tuwien.ac.at/staff/lukasiew/rr0608.pdf.

[21] T. Lukasiewicz and U. Straccia. An overview of uncertainty and vagueness in description logics for the Semantic Web. Technical Report INFSYS RR-1843-06-07, Institut für Informationssysteme, Technische Universität Wien, October 2006.

[22] B. Motik, I. Horrocks, R. Rosati, and U. Sattler. Can OWL and logic programming live together happily ever after? In Proc. ISWC-2006, pp. 501-514, 2006.

[23] B. Motik and R. Rosati. A faithful integration of description logics with logic programming. In Proc. IJCAI-2007, pp. 477-482, 2007.

[24] D. Poole. The independent choice logic for modelling multiple agents under uncertainty. Artif. Intell., 94(1-2):7-56, 1997.

[25] R. Rosati. On the decidability and complexity of integrating ontologies and rules. J. Web Sem., 3(1):61-73, 2005.

[26] R. Rosati. $\mathcal{D} \mathcal{L}+\log$ : Tight integration of description logics and disjunctive datalog. In Proc. KR-2006, pp. 68-78, 2006.

[27] G. Stoilos, G. Stamou, V. Tzouvaras, J. Pan, and I. Horrocks. Fuzzy OWL: Uncertainty and the Semantic Web. In Proc. OWLED-2005, 2005. 
[28] U. Straccia. Transforming fuzzy description logics into classical description logics. In Proc. JELIA2004, pp. 385-399, 2004.

[29] U. Straccia. Towards a fuzzy description logic for the Semantic Web (preliminary report). In Proc. ESWC-2005, pp. 167-181, 2005.

[30] U. Straccia. A fuzzy description logic for the Semantic Web. In E. Sanchez, editor, Fuzzy Logic and the Semantic Web, Capturing Intelligence, chapter 4, pp. 73-90. Elsevier, 2006.

[31] U. Straccia. Uncertainty and description logic programs over lattices. In E. Sanchez, editor, Fuzzy Logic and the Semantic Web, Capturing Intelligence, chapter 7, pp. 115-133. 2006.

[32] U. Straccia. Fuzzy description logic programs. In Proc. IPMU-2006, pp. 1818-1825, 2006.

[33] C. Tresp and R. Molitor. A description logic for vague knowledge. In Proc. ECAI-1998, pp. 361-365, 1998.

[34] W3C. OWL web ontology language overview, 2004. W3C Recommendation (10 Feb. 2004). Available at www.w3.org/TR/2004/REC-owl-features-20040210/. 